\title{
Projectively invariant equations of gravitation
}

\author{
Leonid V. Verozub
}

\begin{abstract}
The paper considers a physical consequences of bimetric geodesically (projectively) invariant equations of gravitation. The correct expression for the energyimpulse tensor of the gravitational field is obtained and with its help the key role of the gravitational defect of mass-energy for the energy of the newborn universe is shown..
\end{abstract}

Keywords Modified theories of gravitation; Cosmology;

leonid.v.verozub

univer.kharkov.ua; lverozub

gmail.com

Tel.: +4917649086679 


\section{Introduction}

The appearance of projectively invariant modification of the classical Einstein equations is caused by a new mathematical result that was obtained many years after the appearance of the Einstein equations. Namely, A. Petrov[3] showed that the Einstein equations are not invariant with respect to a wide class of transformations of the Christoffel symbols and the metric tensor which are not related to coordinate transformation and preserve the equations of motion of the test particles, i.e., geodetic lines. This means that any equations of gravity, based on the hypothesis of the geodesic motion of test particles, must be invariant with respect to these geodesic (projective) transformations. The simplest equations of this kind were considered in [5].

Such a modification entails some important physical consequences, which do not contradict the observations and the study of which is of great interest, since it can help to solve some fundamental problems.

\section{Why do we need projectively invariant equations?}

If we start from Einstein's beautiful hypothesis that test particles in gravitational field move along geodesic lines of some Riemannian spaces $V$, it is natural to expect that the differential equations for finding the metric tensor $g_{\alpha \beta}(x)$ for a given distribution of matter also should be invariant under any transformations at which the geodesic equations remain invariant.

However, the geodesic equations are invariant not only under arbitrary transformation of coordinates (it is rather obvious) but also under geodesic (projective) mappings $\Gamma_{\beta \gamma}^{\alpha}(x) \rightarrow \bar{\Gamma}_{\beta \gamma}^{\alpha}(x)$ of the Christoffel symbols in any fixed coordinate system [9].

In order to see this, we use coordinate time $t=x^{0} / c$ ( $c$ is speed of light) as a parameter along geodesic lines, then the differential equations of a geodesic line are of the form

$$
\ddot{x}^{\alpha}+\left(\Gamma_{\beta \gamma}^{\alpha}-c^{-1} \Gamma_{\beta \gamma}^{0} \dot{x}^{\alpha}\right) \dot{x}^{\beta} \dot{x}^{\gamma}=0,
$$

where $\dot{x}^{\alpha}=d x / d t, \ddot{x}^{\alpha}=d \dot{x}^{\alpha} / d t$. It easy to verify that these equations are invariant under the mapping

$$
\bar{\Gamma}_{\beta \gamma}^{\alpha}(x)=\Gamma_{\beta \gamma}^{\alpha}(x)+\delta_{\beta}^{\alpha} \phi_{\gamma}(x)+\delta_{\gamma}^{\alpha} \phi_{\beta}(x),
$$

where $\phi_{\alpha}(x)$ is any continuously differentiable vector field.

In Riemannian space-time $\phi_{\alpha}(x)$ can be expressed [2,9] in terms of determinants $g$ and $\bar{g}$ of the metric tensors before and after the geodesic mapping of space-time $V$ into $\bar{V}$ as follows:

$$
\phi_{\alpha}=\frac{1}{n+1}\left(\Gamma_{\alpha \gamma}^{\gamma}-\bar{\Gamma}_{\alpha \gamma}^{\gamma}\right)=\frac{1}{2(n+1)} \frac{\partial}{\partial x^{\alpha}} \ln \left|\frac{\bar{g}}{g}\right|
$$

Such mappings of the Christoffel symbols in a given coordinate system induce some mapping not only of the curvature tensors, but also some transformations $g_{\alpha \beta} \rightarrow \bar{g}_{\alpha \beta}$ of the metric tensor which are obtained by solving of the partial differential equation

$$
\bar{g}_{\beta \gamma ; \alpha}(x)=2 \phi_{\alpha}(x) \bar{g}_{\beta \gamma}(x)+\phi_{\beta}(x) \bar{g}_{\gamma \alpha}(x)+\phi_{\gamma}(x) \bar{g}_{\alpha \beta}(x),
$$

where the semicolon denotes a covariant derivative with respect to the metric in $V$. 
It is clear that all solutions of these equation are, in principle, equivalent physically because they define the same system of geodesic lines and consequently, the same gravitational field. However, Einstein's equations are not invariant with respect to such transformations[3] because, for example, the Ricci tensor is transformed under (2) as follows

$$
\bar{R}_{\alpha \beta}=R_{\alpha \beta}-\phi_{\alpha \beta}
$$

where $\phi_{\alpha \beta}=\phi_{\alpha ; \beta}-\phi_{\alpha} \phi_{\beta}$, and $\phi_{\alpha ; \beta}$ is a covariant derivative of $\phi_{\alpha}$ with respect to $x^{\beta}$.

The above have important consequences.

The properties of the classical field are manifested of the properties of the motion of test particles. If these properties of test particles are the same, then the fields are the same.

If the field equations are not invariant with respect to the transformations allowed by the equations of motion of test particles, then they can inadequately describe the physical reality. For example, let's subject the Christoffels to a specific transformation. Then the Ricci tensor will change and the Einstein equations will give a new solutions. If Einstein's hypothesis about the geodesic motion of test particles under the action of gravity is true, then it is also possible that this is gravitation, which the classical equations do not see.

It follows from this fact that in any gravitation equations based on the hypothesis of the motion of test particles along geodesic lines should be invariant with respect to geodesic (projective) mappings.

In the non-relativistic limit only components $\Gamma_{00}^{i}$ are essential. But it follows from (2) that the components $\bar{\Gamma}_{00}^{i}=\Gamma_{00}^{i}$. Therefore, in Newtonian limit, geodesic-invariance is not an essential fact. However this cannot be said about the relativistic case. For this reason it is interesting to explore a theory in which the gravitation equations, as well as the equations of motion of test particles, are geodesic- invariant, i.e., in which geodesic invariance plays the role of gauge invariance.

\subsection{Examples of geodesic non-equivalence}

The most interesting examples of geodesic equivalence of space-time elements give the Friedmann-Lemaître-Robertson-Walker (FLRW) and Schwarzschild solutions.

We use the fact that equations (2) are the necessary and sufficient conditions of the geodesic equivalence [10].

\section{FLRW line element.}

Consider the line elements of a Riemannian space-time $V$ :

$$
d s^{2}=b(t) d t^{2}+a(t) \sigma_{i k}\left(x^{1}, x^{2}, x^{3}\right) d x^{1} d x^{k}
$$

where $a(\mathrm{t})$ and $b(t)$ are arbitrary differentiable functions."

The FLRW meric is a particular case of this metric.

The geodesics of such metric are the same as the ones of the space-time $\bar{V}$ with the line element

$$
\overline{d s}^{2}=B(t) d t^{2}+A(t) \sigma_{i k}\left(x^{1}, x^{2}, x^{3}\right) d x^{k} d x^{k}
$$


where

$$
\begin{gathered}
B(t)=\frac{b(t)}{[1+q a(t)]^{2}}, \\
A(t)=\frac{a(t)}{1+q a(t)},
\end{gathered}
$$

and $q$ is an arbitrary constant.

Indeed, contracting (2) with respect to $\alpha$ and $\beta$, we obtain $\bar{\Gamma}_{\beta \gamma}^{\beta}=\Gamma_{\beta \gamma}^{\beta}+(n+1) \psi_{\beta}$. Consequently,

$$
\psi_{\beta}=\frac{1}{2(n+1)} \frac{\partial}{\partial x^{\beta}} \ln \left|\frac{\operatorname{det} \bar{g}}{\operatorname{det} g}\right|,
$$

which shows that in the case under consideration only 0 -component of $\psi_{\alpha}$ is other than zero.

The useful for us components of the Christoffel symbols of $V$ are given by:

$$
\bar{\Gamma}_{00}^{0}=b^{\prime}(t) / 2 b, \quad \bar{\Gamma}_{11}^{0}=a^{\prime}(t) / 2 b(t)
$$

$$
\bar{\Gamma}_{10}^{1}=a^{\prime}(t) / 2 a(t)
$$

and the same components of $\bar{V}$ are:

$$
\bar{\Gamma}_{00}^{0}=B^{\prime}(t) / 2 B, \quad \bar{\Gamma}_{11}^{0}=-A^{\prime}(t) / 2 B(t),
$$

$$
\bar{\Gamma}_{10}^{1}=A^{\prime}(t) / 2 A(t)
$$

Then eqs. (2) gives the following equations

$$
\begin{gathered}
\frac{A^{\prime}(t)}{A(t)}-\frac{a^{\prime}(t)}{a(t)}=2 \psi_{0}, \\
\frac{B^{\prime}(t)}{B(t)}-\frac{b^{\prime}(t)}{b(t)}=4 \psi_{0}, \\
\frac{A^{\prime}(t)}{B(t)}-\frac{a^{\prime}(t)}{b(t)}=0 .
\end{gathered}
$$

So, $A / a=\exp \left(2 \int \psi(t)_{0} d t\right), B / b=\exp \left(4 \int \psi(t)_{0} d t\right)$ where the integration constants are equal to 1 because at $\psi(t)_{0}=0$ the functions $A(t)=a(t)$ and $B(t)=b(t)$. Consequently, $B(t) / b(t)=(A(t) / a(t))^{2}$, and with (14) we obtain the differential equation

$$
A^{\prime}(x)-A(t)^{2} \frac{a^{\prime}(t)}{a(t)}=0,
$$

which gives (9). Now from previous equation we obtain the function $B(t)$ in the form (8).

On the contrary, if in eq. (2) to set $\psi_{i}=0$ for $\mathrm{i}=1,2,3$, and $\psi_{0}=-1 / 2 \partial \ln (1+$ $q b(t)) / \partial t$, then eqs. (12), (13), and (14) are satisfied. Thus, with this choice of the co-vector field $\psi(x)_{\alpha}$, the line element (6) at $b=-1$ is equivalent to (7). In other words, the both line elements have the same (no-parameterized) equations of motion of test particles. 
The Schwarzschild line element

As another example, we consider here a static centrally symmetric line element

$$
d s^{2}=b(r) d r^{2}+r^{2}\left(d \theta^{2}+\sin ^{2} \theta d \phi^{2}\right)-a(r) d t^{2} .
$$

The Schwarzschild metric is a particular case of this metric. Let us show that this line element in a given coordinate system has common geodesic lines with the element of the form

$$
\overline{d s}^{2}=B(r) d r^{2}+F(r)^{2}\left(d \theta^{2}+\sin ^{2} \theta d \phi^{2}\right)-A(r) d t^{2},
$$

where $A(x), B(x)$ and $F(x)$ are functions of $x^{\alpha}$, depending on a continuous parameter.

The Christoffel symbols for (16) is given by

$$
\begin{array}{ll}
\Gamma_{r r}^{r}=\frac{1}{2} \frac{b^{\prime}(r)}{b(r)}, \Gamma_{r \theta}^{\theta}=\frac{1}{r}, \Gamma_{r \phi}^{\phi}=\frac{1}{r}, \Gamma_{r t}^{t}=\frac{1}{2} \frac{a^{\prime}(r)}{a(r)}, & \Gamma_{\theta \theta}^{r}=-\frac{r}{b(r)}, \\
\Gamma_{\theta \phi}^{\phi}=\frac{\cos \theta}{\sin \theta}, \Gamma_{\phi \phi}^{r}=-\frac{r \sin ^{2} \theta}{b(r)}, \Gamma_{\phi \phi}^{\theta}=-\sin \theta \cos \theta, & \Gamma_{t t}^{r}=\frac{1}{2} \frac{a^{\prime}(r)}{b(r)} .
\end{array}
$$

The Christoffel symbols for 17 are:

$$
\begin{gathered}
\bar{\Gamma}_{r r}^{r}=\frac{1}{2} \frac{B^{\prime}(r)}{B(r)}, \bar{\Gamma}_{r \theta}^{\theta}=\frac{F^{\prime}(r)}{F(r)}, \bar{\Gamma}_{r \phi}^{\phi}=\frac{F^{\prime}(r)}{F(r)}, \\
\bar{\Gamma}_{r t}^{t}=\frac{1}{2} \frac{A^{\prime}(r)}{A(r)}, \bar{\Gamma}_{\theta \theta}^{r}=-\frac{F(r) F^{\prime}(r)}{B(r)}, \\
\bar{\Gamma}_{\theta \phi}^{\phi}=\frac{\cos \theta}{\sin \theta}, \bar{\Gamma}_{\phi \phi}^{r}=-\frac{F(r) F^{\prime}(r) \sin A p J^{2} \theta}{B(r)}, \\
\bar{\Gamma}_{\phi \phi}^{\theta}=-\sin \theta \cos \theta, \bar{\Gamma}_{t t}^{r}=\frac{1}{2} \frac{A^{\prime}(r)}{B(r)},
\end{gathered}
$$

where a prime here and later denotes a derivative with respect to $r$.

In view of this, Levi-Chevita equations (2) yields:

$$
\begin{aligned}
\frac{B^{\prime}(r)}{B(r)}-\frac{b^{\prime}(r)}{b(r)}=4 \psi_{r}(r), F(r) F^{\prime}(r) b(r)-r B(r) & =0, \\
& A^{\prime}(r) b(r)-a^{\prime}(r) B(r)=0,
\end{aligned}
$$


$\frac{F^{\prime}(r)}{F(r)}-\frac{1}{r}=\psi_{r}(r), \frac{A^{\prime}(r)}{A(r)}-\frac{a^{\prime}(r)}{a(r)}=2 \psi_{r}(r)$, $\psi_{\theta}(r)=\psi_{\phi}(r)=\psi_{t}(r)=0$

According to (10) the function $\psi_{r}(r)$ can be written as

$$
\psi_{r}=\partial \ln \chi / \partial r
$$

where

$$
\chi(r)=\left(\frac{\bar{g}}{g}\right)^{1 / 2(n+1)} .
$$

Consequently,

$$
\begin{aligned}
B & =b \chi^{4} ; \quad A=a \chi^{2} ; \quad F=\chi ; \\
A^{\prime} & =a^{\prime} \chi^{4} ;\left(F^{2}\right)^{\prime}=2 r \chi^{4}
\end{aligned}
$$

Formulas for the function $F(r)$ are compatible only if the functions $\chi(r)$ are the solution of the differential equations

$$
r \chi^{\prime}(r)+\chi(r)-\chi(r)^{3}=0
$$

which yields

$$
\chi(r)=\left(1+k r^{2}\right)^{1 / 2}
$$

where $k$ is an arbitrary constant.

As a result, formulas which express the $A(r), B(r)$, and $F(r)$ by $a(r)$ and $b(r)$ are given by

$$
\begin{aligned}
& A(r)=\frac{a(r)}{1+k r^{2}}, B(r)=\frac{b(r)}{\left(1+k r^{2}\right)^{2}}, \\
& F(r)=\frac{r}{\left(1+k r^{2}\right)^{1 / 2}} .
\end{aligned}
$$

These functions with the arbitrary parameter $k$ define the same geodesic lines.

\section{Generalization of Einstein's vacuum equations}

The study of the possibility of a geodesically (projectively) invariant generalization of the Einstein equations shows [5] that it is necessary to use the projectively invariant Thomas symbols [2,9] instead of Christoffel symbols. However, in this case, constructing a projectively invariant generalization of the Ricci tensor is possible only within the framework of a certain bimetric theory. We have to assume that in the theory of gravitation it is necessary to use Minkowski space in addition to a Riemann space, which Rosen [4] had long suggested.

The simplest of such equations are [5]

$$
\nabla_{\alpha} B_{\beta \gamma}^{\alpha}-B_{\beta \delta}^{\epsilon} B_{\epsilon \gamma}^{\delta}=0
$$


where $\nabla_{\alpha}$ denotes a covariant derivative in Minkowski space,

$$
\begin{gathered}
B_{\beta \gamma}^{\alpha}=\Pi_{\beta \gamma}^{\alpha}-\stackrel{\circ}{\Pi}_{\beta \gamma}^{\alpha}, \\
\stackrel{\circ}{\Pi}_{\alpha \beta}^{\gamma}=\stackrel{\circ}{\Gamma}_{\alpha \beta}^{\gamma}-(n+1)^{-1}\left[\delta_{\alpha}^{\gamma} \stackrel{\circ}{\Gamma}_{\beta}+\delta_{\beta}^{\gamma} \stackrel{\circ}{\Gamma}_{\alpha}\right]
\end{gathered}
$$

are Thomas symbols for Minkowski space in the used coordinate system.

They are some bimetric equations. For this reason, coordinate systems can be chosen arbitrarily in Minkowski space and consequently, coordinates have an operational meaning.

These equations have similarities to vacuum equations of classical electrodynamics in Minkowski space:

$$
\mathcal{F}_{\alpha ; \gamma}^{\gamma}=0, \quad \mathcal{F}_{\alpha \beta}=\partial_{\alpha} A_{\beta}(x)-\partial_{\beta} A_{\alpha}(x),
$$

which are invariant relative to mappings of 4-potentials: $A_{\alpha} \rightarrow A_{\alpha}+\phi(x)_{\alpha}$. However, eqs. (25) contains the right-hand side which expresses self-interaction of gravitation.

When we select the covariant gauge conditions in the form $Q_{\alpha}=\Gamma_{\alpha \beta}^{\beta}-\stackrel{\circ}{\Gamma \beta}_{\alpha \beta}^{\beta}=0$, equations (25) coincide with the classical vacuum Einstein equations $R_{\alpha \beta}=0$.

In other words, the classic vacuum Einstein's equations coincide with equations (25) at the gauge conditions $Q_{\alpha}=0$.

\section{Spherically symmetric gravity}

\section{Motivation}

To better understand the difference between general relativity (GR) and the suggested theory, we first analyze the process of the solutions of the vacuum Einstein's equations in the gravity field of a point mass $M$, and after that compare it with the process of the solving this problem by considered here equations.

\subsection{Spherically symmetric solution in GR}

The solution of Einstein's equations are defined up to an arbitrary coordinate transformations. Based on this fact, we can choose the coordinate system (CS) so that the line element would have a simple form. The selected system can be characterized only by the words: "It is such that the line element in this system has the following form". The selected CS can not be characterized by an operational way.

Fortunately, in this case, it is obvious that space-time is flat at infinite distance from the source of gravity. Therefore, the coordinate system can be selected so that at infinity it coincides with spherical coordinates of Minkowski space, that is, in general the line element is assumed to have the form:

$$
d s^{2}=C(r) d x^{0^{2}}-A(r) d r^{2}-B(r)\left[d \theta^{2}+\sin ^{2} \theta d \varphi^{2}\right]-D(r) d r d t .
$$

If we substitute (28) to eqs. $R_{\alpha \beta}=0$, we get ordinary differential equations for finding these functions. 
It turns out that the number of equations, is not enough to uniquely solve this problem. It is a consequence of the Bianchi identities. To find a definite solution, further specification of the coordinate system is necessary.

This is achieved by the use of additional restrictions on the coefficients of the line element. Depending on the selected conditions different solutions will be obtained. The most popular are the conditions: $D(r)=0, B(r)=r^{2}$.

It should be also assumed (which is quite natural) that at infinity

$$
\lim _{r \rightarrow \infty} A=1, \lim _{r \rightarrow \infty} C=1 .
$$

But this is not all. The resulting solution also depends on a constant which can be found only from physical considerations. It should be assumed that in the nonrelativistic limit the equations of motion of a test particle must coincide with the equations of Newtonian mechanics. Then this constant is uniquely determined. It is the Schwarzschild radius $r_{g}=2 G M / c^{2}$ where $G$ is the gravitational constant and $c$ is speed of light. Finally, the line element is

$$
d s^{2}=C d x^{0^{2}}-A d r^{2}-r^{2}\left[d \theta^{2}+\sin ^{2} \theta d \varphi^{2}\right]
$$

where

$$
A(r)=\frac{1}{C(r)}, \quad C(r)=1-\frac{r_{g}}{r} .
$$

This solution was obtained by Weyl and Droste [50], and named after Schwarzschild, who first got the exact solution of Einstein's equations though in a different form.

4.2 Spherically symmetric solution of the equations under consideration.

Section 6 contains the substantiation of the non-trivial fact, first investigated by Rosen, what is the physical meaning of the coexistence of two spaces in the theory of gravitation. In this section, we'll just take this opportunity and explore the gravitational equations in Minkowski space in orthogonal coordinates.

Assuming that the Lagrangian describing motion of particles is invariant under the mapping $t \rightarrow-t$, it can be written in the form

$$
L=-m c\left[C c^{2}-A \dot{r}^{2}-B\left(\dot{\theta}^{2}+\sin ^{2} \theta \dot{\varphi}^{2}\right)\right]^{1 / 2},
$$

where $A, B$ and $C$ are the functions of the radial coordinate $r$.

The associated line element of space-time in PFRs is

$$
d s^{2}=C d x^{0^{2}}-A d r^{2}-B\left(d \theta^{2}+\sin ^{2} \theta \varphi^{2}\right) .
$$

We can to look for the solution of equations (25) in Minkowski space on the base of Lagrangian ( 29).

To solve equations (25) it is very convenient to use the fact that when choosing the gauge condition $D_{\alpha}=\Gamma_{\alpha \beta}^{\beta}-\stackrel{\circ}{\Gamma}_{\alpha \beta}^{\beta}=0$ of the equation, the modified vacuum equations coincide with the Ricci equations $R_{\alpha \beta}=0$.

So, we should the system

$$
R_{\alpha \beta}=0 ; D_{\alpha}=0
$$


At the condition

$$
\lim _{r \rightarrow \infty} A=1, \lim _{r \rightarrow \infty}\left(B / r^{2}\right)=1, \lim _{r \rightarrow \infty} C=1 .
$$

Unlike general relativity, we cannot reduce the number of unknown functions in the Lagrangian because the coordinate system is already specified.

The used gauge condition is (30) yield one equation:

$$
B^{2} A C=r^{4} .
$$

It allows us to exclude the function $A$. Then the equations $R_{11}=0$ and $R_{00}=0$ are:

$$
\begin{gathered}
-2 B C^{\prime}+2 r B^{\prime} C^{\prime}+r B C^{\prime \prime}=0, \\
-4 B C B^{\prime}+r C B^{\prime 2}-2 B C^{\prime}+2 r B B^{\prime} C^{\prime}+2 r B C B^{\prime \prime}+r B^{2} C^{\prime \prime}=0 .
\end{gathered}
$$

Because of equality ( 33 ) the sum of three terms in (34) is equal to zero, and we obtain a differential equation for the function $B$ :

$$
2 r B B^{\prime \prime}+r B^{2}-4 B B^{\prime}=0 .
$$

A general solution of this equation can be written as $B=a\left(r^{3}+\mathcal{K}^{3}\right)^{2 / 3}$ where $a$ and $\mathcal{K}$ are some constants. The constant $a=1$, which can be seen from condition (31).

The function $C$ can be found from differential equation (33) in the form

$$
C^{\prime \prime}+2 \frac{r B^{\prime}-B}{r B} C^{\prime}=0
$$

where $\left(r B^{\prime}-B\right) / r B=\left(r^{3}-\mathcal{K}^{3}\right) /\left(r^{4}+r \mathcal{K}^{3}\right)$. A general solution of this equation is $C=b-\mathcal{Q} / B^{1 / 2}$ where $b$ and $\mathcal{Q}$ are a constant. It follows from (31) that $b=1$.

Thus, in the spherical coordinate system the functions $A, B$ and $C$ are given by:

$$
A=\frac{r^{4}}{f^{4}(1-\mathcal{Q} / f)}, B=f^{2}, C=1-\frac{\mathcal{Q}}{f} .
$$

where

$$
f=\left(r^{3}+\mathcal{K}^{3}\right)^{1 / 3}
$$

In non-relativistic limit the radial component of the equations of the motion of a test particle (1) takes the form $\ddot{x}^{r}=-c^{2} \Gamma_{00}^{r}$, where $\Gamma_{00}^{r}=C^{\prime} / 2 A=r^{4} C^{\prime} / f^{4} C$. Therefore, to obtain the Newton gravity law it should be supposed that at large $r$ the function $f(r)$ coincides with $r$, and $\mathcal{Q}=r_{g}=2 G M / c^{2}$ is the classical Schwarzschild radius.

At the given constant $\mathcal{Q}$ allowable solutions (37) are obtained with arbitrary constant $\mathcal{K}$. In particular, if we set $\mathcal{K}=0$, then the line element (30) of the space-time in PFRs coincides with the Droste-Weyl solution of Einstein's equations [50],i.e., general accepted solution in GR which has an event horizon at $r=r_{g}$ :

$$
d s^{2}=-\frac{d r^{2}}{\left(1-r_{g} / r\right)}-r^{2}\left[d \theta^{2}+\sin ^{2} \theta d \varphi^{2}\right]+\left(1-r_{g} / r\right) d x^{0^{2}} .
$$

If we set $\mathcal{K}=\mathcal{Q}$, the line element coincides with the original solution [51] of Einstein's equations by Schwarzschild:

$$
d s^{2}=-\frac{f^{\prime 2} d r^{2}}{\left(1-r_{g} / f\right)}-f^{2}\left[d \theta^{2}+\sin ^{2} \theta d \varphi^{2}\right]+\left(1-r_{g} / f\right) d x^{0^{2}},
$$


where $f=\left(r_{g}^{3}+r^{3}\right)^{1 / 3}$. This solution has no event horizon and no singularity in the center.

Indeed, for the motion of a particle in the plane $\theta=\pi / 2$ laws of conservation of energy $E$ and momentum $J$ take place:

$$
\dot{r} \frac{\partial L}{\partial \dot{r}}-\dot{\varphi} \frac{\partial L}{\partial \dot{\varphi}}-L=E, \quad \frac{\partial L}{\partial \dot{\varphi}}=J
$$

It follows from this fact that the equations of the motion are of the form

$$
\begin{gathered}
\dot{r}^{2}=\frac{c^{2} C^{2} \bar{f}^{4}}{\bar{r}^{4}}\left[1-\frac{C}{\bar{E}^{2}}\left(1+\frac{\bar{J}^{2}}{\bar{f}^{2}}\right)\right], \\
\dot{\varphi}=\frac{\bar{J}}{\bar{E}} \frac{C c r_{g}}{f^{2}}
\end{gathered}
$$

where $(t, r, \varphi)$ are the spherical coordinates, $\dot{r}=d r / d t, \dot{\varphi}=d \varphi / d t, \bar{E}=E / m c^{2}$, $\bar{J}=J / r_{g} m c$.

The radial velocity of freely falling particle is given by the equation

$$
v=c\left[\frac{C^{2} f^{4}}{r^{4}}(1-C)\right]^{1 / 2}
$$

Fig. 3.6.1 shows the plot of the velocity of a test particle as a function of the distance $r / r_{g}$ from the center of

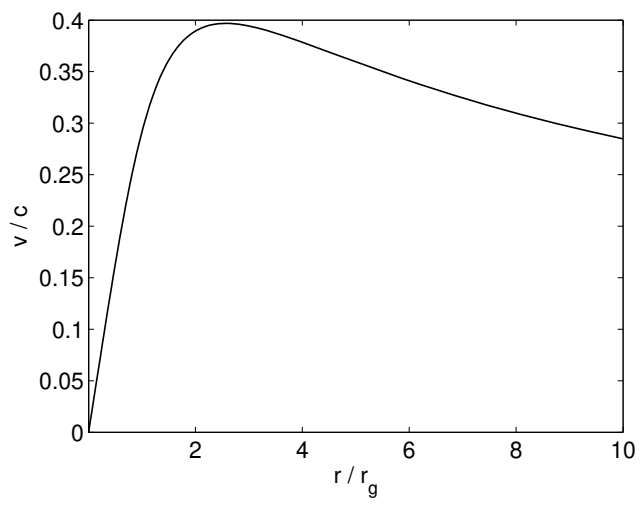

Fig. 1 The velocity of a freely falling particle (in units of $c$ ) as the function of the distance from the center.

instruments.

Suppose, as it is usually believed, (40) can be transformed to the form (39) by an appropriate local coordinate transformation $r \rightarrow r^{\prime}$ and $t \rightarrow t^{\prime}$. The correctness of the equivalence of the original Schwarzschild's solution and the solution of the Weyl-Droste is not completely obvious [50]. But suppose that these solutions within the framework of general relativity are indeed equivalent.

These new coordinates have not an operational meaning, and after an appropriate transformation of the physical 3-space and time intervals, the same physical results will 
be obtained from (39) as from (40). (Just as in classical electrodynamics in arbitrary coordinates). In particular, since the line element (40) at $\mathcal{K}=\mathcal{Q}$ has no the event horizon and the singularity in the center in spherical coordinates, it does not contain it in other coordinate systems.

From our point of view, the functions found ( 37 ) do not have, by themselves, the physical sense, because they are defined only up to arbitrary geodesic mappings. For a different choice of the gauge condition these functions, in general, will be different. Physical meaning have only gauge-invariant functions $B_{\beta \gamma}^{\alpha}(x)$. In the spherical coordinates, they have the following form:

$$
\begin{array}{r}
B_{r r}^{r}=\frac{1}{2} \frac{A^{\prime}}{A} ; \quad B_{\theta \theta}^{r}=\frac{1}{2} \frac{2 r A-B^{\prime}}{A} ; \\
B_{\phi \phi}^{r}=-\frac{1}{2} \frac{2 r A-B^{\prime}}{A} \sin ^{2} \theta ; \\
B_{t t}^{r}=\frac{1}{2} \frac{C^{\prime}}{A} ; \quad B_{t r}^{t}=\frac{1}{2} \frac{C^{\prime}}{C} \\
B_{\theta r}^{\theta}=\frac{1}{2} \frac{2 B-B^{\prime} r}{r B} \quad ; B_{\phi r}^{\phi}=\frac{2 B-B^{\prime} r}{r B} .
\end{array}
$$

Fortunately, the components of these functions which do not depend on the angular variables coincide with Christoffel symbols. This means that these components of the Christoffels symbols are equvalent to the corresponding components of projectively invariant tensor $B_{\beta \gamma}^{\alpha}(x)$. Consequently, the use of the Christoffel symbols is quite correct in a spherically symmetric gravitational field at the. And for this reason, solutions of Einstein's equations give correct results at distances much larger than the Schwarzschild radius.

It must be noted that by using the above results we can find also the gravitational field inside any spherically-symmetric shell [5].

\section{Equations inside matter}

A geodesically invariant generalization of Einstein's equations in matter is also possible ([5]). These equations has the form

$$
\nabla_{\alpha} B_{\beta \gamma}^{\alpha}-B_{\beta \delta}^{\epsilon} B_{\epsilon \gamma}^{\delta}=k\left(T_{\alpha \beta}-\frac{1}{2} \hat{g}_{\alpha \beta} T\right)
$$

where a gauge-invariant tensor

$$
\hat{g}_{\alpha \beta}=g_{\alpha \beta}-(n+1)^{-1} Q_{\alpha} Q_{\beta} .
$$

and $Q_{\alpha}=\frac{1}{n+1}\left(\Gamma_{\alpha \beta}^{\beta}-\stackrel{\circ}{\Gamma^{\beta}}{ }_{\alpha \beta}\right)$.

The classic Gilbert-Einstein equations

$$
R_{\alpha \beta}=k \cdot\left(T_{\alpha \beta}-\frac{1}{2} g_{\alpha \beta} T\right)
$$

are the equations (45) at the gauge conditions $Q_{\alpha}=0$.

The gravitational equations in matter are very difficult to verify, unlike the vacuum equations, which are well supported by numerous observations. 
In addition, it is unclear what physical meaning the requirement of geodesic invariance inside of a matter.

For these reasons, we do not use these equations.

\section{The physical meaning of bimertrism in the theory of gravity}

6.1 Space-time relativity with respect to measuring instruments

Newton's idea of the absoluteness of physical space has always been criticized by many thinkers. Alternative point of view has been confirmed by Einstein's gravitational theory, which shows, basing on observations, that the properties of space-time are interconnected with the properties of matter.

However, there is also another kind of relativity of space and time. Awareness of this fact is a merit of Herman Helmholtz and Henri Poincaré.

Helmholtz was the first who realized the importance of the measurement process by imaginary measuring instruments for establishing the properties of the geometry of space. Poincaré developed these ideas. He convincingly proved that there is a vicious circle: in order to characterize the geometry of space and time we need measuring devices (rulers and clocks), but in order to characterize properties of the measuring instruments we need to know the geometry of space and properties of time. Consequently, he says, it makes no sense to say that this or that geometry is correct. The choice of geometry is only a matter of convenience. It follows that the validity of this or that geometry can not be proved by experience.

The results of Poincaré was misinterpreted by philosophers and interpreted as conventionalism. They have a deeper meaning. Hans Reichenbach was closest to understanding this. Here is an excerpt from his book "The Philosophy of Space and Time" [1]:

"Mathematics proves that every geometry of the Riemannian kind can be mapped upon another one of the same kind. In the language of physics this means the following:

"Theorem 8:... Given a geometry G' to which the measuring instruments conform, we can imagine a universal force $F$ which affects the instruments in such a way that the actual geometry is an arbitrary geometry G, while the observed deviation from $G$ is due to a universal deformation of the measuring instruments. $"$...

"Theorem 8 shows all geometries to be equivalent; it formulates the principle of the relativity of geometry. It follows that it is meaningless to speak about one geometry as the true geometry. We obtain a statement about physical reality only if in addition to the geometry $\mathrm{G}$ of the space its universal field of force $\mathrm{F}$ is specified. Only the combination

$$
G+F
$$

is a testable statement."

All that has been said above can certainly be repeated for space-time. And of course, the words about the force $\mathrm{F}$ are not to be taken literally.

Based on all the above, we can formulate the following statement: 
To describe the geometry of space-time, we need to know the properties of measuring instruments. However, to describe the measuring instruments, we need, strictly speaking, the geometry of space-time. Thus, strictly speaking, space-time geometry and properties of the instrument do not have a meaning in itself.

Only a combination of "measuring instruments + space-time geometry" has a physical, verifiable by experiment meaning.

This is how the result of Poincaré should be understood.

By itself, this result has only philosophical nature and does not provide any guidance for physics. That is why it was not implemented in physical theory.

In order to make a step towards applying this result, it should be taken into account that the properties of space-time and the properties of the measuring devices are naturally interconnected with each other, if we describe physical phenomena in a certain frame of reference.

For this reason, we formulate and examine here the Poincaré-Reichenbach idea in the following form:

Only a combination of "reference frame + space-time geometry" has a physical, verifiable by experiment meaning.

Let us first consider the inertial frame of reference (IRF).

There are various definitions of an inertial frame of reference, but they all recognize that in the absence of force fields, space-time is pseudo-Euclidean. Therefore, it can be postulated that the combination "inertial frame + Minkowski space" is correct.

By itself, IRF is a some set of test particles with clocks. The clock is a periodic process that measures the length of its own world line.

The coordinate system is considered here as a method of parametrization of events in space-time. The parametrization is arbitrary, and physical results, of course, should not depend on a choice of the coordinate system.

We suppose that all definitions must have operational meaning. We attach great importance to this condition, since otherwise the definitions have no physical meaning for us.

6.2 Space-time in "proper reference frames"

According to the above, we postulate that the set "inertial reference frame + pseudoEuclidean geometry" is the correct choice and we will try to find the space-time geometry in non-inertial reference frames.

We consider a non-inertial reference frame (NRF) as a some set of particles with clocks moving under the action of a force field in an inertial reference frame (IRF).

Consider NRF, whose reference body is formed by a set of identical point masses $m$ moving under the action of a force field $F(x)$, given in Minkowski space of an IRF. This reference frame will be named the proper reference frame (PRF) of the field $F(x)$.

Any NFR is the PRF of some force field.

Such a system can be described by the field of 4 -velocities $\tau^{\alpha}(x)=d x^{\alpha} / d \sigma$ given in the IRF, i.e., in Minkowski space.

This definition of guarantees us that all physical quantities will have an operational, physical meaning.

The motion of these points is defined a congruence of world lines of the particles of the reference body in the Minkowski space. 
On the basis of foregoing, we try to understand what should be the space-time geometry in PRFs of a force field $F(x)$. In other words, we want to find the line element of space-time for an observer located in a PRF which moves in the force field $F(x)$ together with the particles of the reference body of this PRF.

Of course, the observer may not always be in the PRF literally. For example, it is impossible in the reference frame, whose reference body is formed by electric charges moving in an electromagnetic field. However, in this case also, the problem allows a limited study, since the reference body can be formed by ions and each ion can be considered as an atomic clock, whose indications can be readout.

The existence of a connection between space-time metric in IRFs and PRFs can be shown by analysis of a simple and well-known Einstein's elevator considered from an other point of view.

Disregarding the rotation of the Earth, the reference frame, rigidly bound with the Earth surface, can be considered as the inertial one.

An observer, located in this frame, can describe a changing relative distance between the dust particles, free-falling to the Earth from the space, as a result of the existence of a gravitational force $F(x)$ of the Earth in the framework non-relativistic mechanics. He doesn't need non-Euclidean geometry.

However, consider another observer, who is located in the PRF of the force $F(x)$. Let us assume that the dust is so thick that he is unable to see the Earth and the stars, and forgets about them for a moment. Then the observer will assume that the dust particles, as well as himself, are at rest in some physical space. In fact, he considers the dust particles as points of his physical space, because he sees nothing else.

This opinion of the observer indicates that Newton was wrong when he spoke of the absolute space. Moreover, he observes the changing of the relative distance between the dust particles, which indicates that space is "compressed" in some direction. How can we explain this fact? We do not know what the observer will think, but for us, there is apparently the only explanation. This is a consequence of geodesic deviation in non-Euclidean space-time.

Our measuring instruments will show that the change in the relative distance between the particles will be the same for both observers. Thus if the first observer, located in the IRF, can consider space-time as flat, the second observer, located in a $\mathrm{PRF}$ of the force field, has to consider space-time as Riemannian with curvature other than zero.

The relative acceleration of free particles in a falling elevator has been regarded, in particular, as a manifestation of the curvature of space-time. However, one should pay attention to the fact that, from the point of view of an observer on Earth's surface, it occurs in a non-inertial frame of reference since the elevator moves under action of a force field - of gravitation.

To make this conclusion more convincing, we can also refer to the situation in cosmology.

It is well known that an observer on Earth, who believes that his reference system is inertial, may consider expanding the Universe as dust-like matter in the flat space-time on the basis of Newtonian mechanics.

On the other hand, the observer can consider himself to be in the frame of reference whose body is formed by distant galaxies. In this frame, galaxies are at rest. They are points of his physical space and, of course, have no acceleration. In this case, he is compelled to explain the change in the relative distance between galaxies by the deviation of geodesics in non-Euclidean space-time [20]. 
Let us return now to the goal of finding the space-time element in the NRF. ${ }^{1}$

The reference body (RB) of a reference frame is supposed to be formed by identical point masses $m$. If an observer in this reference frame is at rest, his world line coincides with the world line of some point of the reference body. It is obvious for this observer that the accelerations of the point masses forming his reference body are equal to zero. That is, if the line element of space-time in an inertial reference frame is denoted by $d \sigma$ and $u^{\alpha}=d x^{\alpha} / d \sigma$ is the field of 4 - velocity of the point masses forming the reference body, then the absolute derivative of $u^{\alpha}$ is equal to zero: ${ }^{2}$

$$
D u^{\alpha} / d \sigma=0 \text {. }
$$

(We mean that an arbitrary coordinate system is used.)

The same should occur in the PRF. That is if the line element of space-time in the PFR is denoted by $d s$, the 4 -velocity vector $\zeta^{\alpha}=d x^{\alpha} / d s$ of the point-masses forming the reference body of the PRF should satisfy the equation

$$
D \zeta^{\alpha} / d s=0
$$

The equations (48) uniquely determines the fundamental metric form in NRFs.

Indeed, these differential equations of world lines are at the same time the Lagrange equations describing, in Minkowski space, the motion of the point masses forming the reference bodies of the PRF. Therefore, eq. (48) can be derived from a Lagrange action $S$ by the principle of the least action. The equations of the geodesic lines can be obtained from a line element $d s=k d S$, where $k$ is a constant, $d S=\mathcal{L}(x, \dot{x}) d t$, and $\mathcal{L}(x, \dot{x})$ is a Lagrange function describing, in Minkowski space, the motion of identical point masses $m$ forming the body reference of the non-inertial reference frame PRF. The constant $k$ is equal to $-(m c)^{-1}$, as follows from the analysis of the case when the frame of reference is inertial when $\mathcal{L}(x, \dot{x})=-m c d s$ at the signature $\left(+-\right.$ - $\left.^{-}\right)$.

Thus, if we proceed from relativity of space and time in the Berkeley-Leibniz-MachPoincaré (BLMP) sense, then the line element of space-time in PRFs can be expected to have the following form $[5,6]$

$$
d s=-(m c)^{-1} d S(x, d x) .
$$

Therefore, properties of space-time in PRFs are entirely determined by properties of the used frames in accordance with relativity idea of relativity of space and time.

\section{Example 1. Electromagnetic field}

The reference body consists of noninteracting electric charges in an electromagnetic field. In a Cartesian coordinate system the action describing the motion of the particles in an IRF can be written as follows [4]:

$$
S=\int\left(-m c^{2}\left(1-v^{2} / c^{2}\right)^{1 / 2}-\frac{e}{c} A_{\alpha}(x) d x^{\alpha} / d t\right) d t
$$

where $A_{\alpha}$ is the 4 -potential, $e$ is the charge of the particles ${ }^{3}$

\footnotetext{
1 The first, unsuccessful, attempt to do this was made in [?]

2 We use notations and definitions, following the Landau and Lifshitz book [4].

3 As usual, in this paper Greek letters run from 0 to 3 , and Latin - from 1 to 3 .
} 
For the given reference frame

$$
d s=d \sigma+\frac{e}{m c} A_{\alpha} d x^{\alpha},
$$

where $d \sigma$ is the line element of space-time in the IRF. It is a Finslerian metric.

Of course, such a frame of reference is not similar to the accelerated reference frame formed by neutral particles. However, this does not prevent its theoretical analysis, assuming that the reference body is formed by identical ions. They can be regarded as atomic clocks that are almost unaffected by accelerations.

Based on the fact that the clocks measure the length of its own world line, one can find the time interval in such PRFs.

Evidently,

$$
d T=\stackrel{0}{d T}+\frac{e}{m c^{2}} A_{\alpha} d \dot{x}^{\alpha} d t
$$

where $d T=d s / c$ and $d T^{0}=d \sigma / c$ are proper time intervals in the PRF and IRF, correspondingly.

The following two cases are of interest:

A) The reference body consists of noninteracting electric charges in a constant homogeneous electric field $E$ directed along the axis $x$. According to (51)

$$
d T / d t=1-\frac{e}{m c^{2}} \varphi=1+\frac{e}{m c^{2}} E x,
$$

where $\varphi=A_{0}$, and $E$ is the electric field strength. Because the electric force $e E=$ maw, where $w$ is the acceleration with respect to the IFR, this result is equivalent to the well known one:

$$
d T / d t=1+\frac{w x}{c^{2}} .
$$

Therefore, this can be regarded as a manifestation of the Finslerian nature of spacetime. This geometry is not a kinematic effect, but it is caused by the existence of a force field in the laboratory inertial frame.

A similar situation also takes place in the magnetic field below.

The same result is obtained for the PRF of the homogeneous field of the Earth, a reference body of which is formed by particles free-falling in the field. The reason is that the replacement $e \varphi$ by the gravitational potential leads to the same equation of the motion of test particles as the equations for charges.

$B$ ) The reference body consists of non-interacting electric charges in a constant homogeneous magnetic field $H$ directed along the axis $z$. The Lagrangian describing the motion of the particles can be written as follows landau:

$$
L=-m c^{2}\left(1-v^{2} / c^{2}\right)^{1 / 2}-\left(m \Omega_{0} / 2\right)(\dot{x} y-x \dot{y}),
$$

where $\dot{x}=d x / d t, \dot{y}=d x / d t$, and $\Omega_{0}=e H / 2 m c$.

The points of such a system rotate in the plane $x y$ around the axis $z$ with the angular frequency

$$
\omega=\Omega_{0}\left[1+\left(\Omega_{0} r / c\right)^{2}\right]^{-1 / 2},
$$

where $r=\left(x^{2}+y^{2}\right)^{1 / 2}$. The linear velocity of the BR points tends to $c$ when $r \rightarrow \infty$. 
For the given PRF

$$
d s=d \sigma+\left(\Omega_{0} / 2 c\right)(y d x-x d y) .
$$

In the above PRFs $d s$ is of the form

$$
d s=\mathcal{F}(x, d x),
$$

where $\mathcal{F}(x, d x)=d \sigma+f_{\alpha}(x) d x^{\alpha}, f_{\alpha}$ is a vector field. Therefore, $\mathcal{F}(x, d x)$ is a homogeneous function of the first degree in $d x^{\alpha}$, that is, $\mathcal{F}(x, k y)=k \mathcal{F}(x, y)$. Thus the space-time in the above NRFs are Finslerian [9].

By using the identities

$$
\mathcal{F}(x, y)=\frac{\partial F(x, y)}{\partial y^{\alpha}} \xi^{\alpha} ; \quad \frac{\partial^{2} \mathcal{F}(x, y)}{\partial y^{\alpha} \partial y^{\beta}}=0,
$$

function $\mathcal{F}^{2}(x, y)$ can be written as

$$
\mathcal{F}(x, y)^{2}=G_{\alpha \beta}(x, y) y^{\alpha} y^{\beta},
$$

where

$$
G_{\alpha \beta}(x, y)=\frac{1}{2} \frac{\partial^{2} \mathcal{F}(x, y)^{2}}{\partial y^{\alpha} \partial y^{\beta}}
$$

is an analog of the fundamental metric tensor of the Riemannian space-time. Consequently, the modulus of the vector $y^{\alpha}$ in point $x^{\alpha}$ is $\|y\|=\mathcal{F}(x, y)=\left[G_{\alpha \beta}(x, y) y^{\alpha} y^{\beta}\right]^{1 / 2}$.

For $d s$ of the form (57) we have

$$
G_{\alpha \beta}(x, y)=\eta_{\alpha \beta}+f_{\alpha} f_{\beta}+\left(\eta_{\alpha \beta}-\tau_{\alpha} \tau_{\beta}\right) f_{\sigma} \tau^{\sigma}+f_{\alpha} \tau_{\beta}+f_{\beta} \tau_{\alpha},
$$

where $\tau^{\alpha}=d x^{\alpha} / d \sigma$.

A covariant vector in the Finslerian space-time, therefore, can be defined as

$$
\stackrel{*}{y}_{\alpha}=\mathcal{F}(x, y) \frac{\partial \mathcal{F}(x, y)}{\partial y^{\alpha}}=G_{\alpha \beta}(x, y) y^{\alpha} .
$$

The orthogonality of vectors $y^{\alpha}$ and $y_{1}^{\alpha}$ can be defined by the equality $\stackrel{*}{y}_{\alpha} y_{1}^{\alpha}=0$. It must be noted that the orthogonality of two vectors is not symmetric.

Let us find the connection between an element of time in the PRF and the IRF.

It follows from the Stokes theorem that in this case the modulus $A$ of the potential $A_{i}$ of a particle at the distance $r$ from the center of the orbit of the reference body is equal $A=H r / 2$, which shows that $A$ is a modulus of the 3 -vector $\vec{A}=\frac{1}{2} \vec{B} \times \vec{r}$, and the $\vec{A}$ is directed tangentially to the orbit circle. For this reason, according to (51),

$$
d T / d t=1-\frac{e}{m c^{3}} \frac{H r^{2} \omega}{2}=1-\frac{F_{l} r}{2 m c^{2}},
$$

where $\omega=d \varphi / d t$ is the angular velocity of the body reference, and $F_{l}=e H r \omega / c$ is Lorentz force. Since the centrifugal force $F_{c}=F_{l}=m w_{c}$, where $w_{c}$ is the centrifugal acceleration, we arrive at the conclusion that this result is equivalent to the well known one:

$$
d T / d t=1-\frac{\omega^{2} r^{2}}{2 c^{2}}
$$

This result coincides with the result for the rotating disk due to the fact that the motion of points on the disk can be described by a similar Lagrangian.

We again see that the Firsler character of space-time in the NRF is due to the force field specified in the IRF. 
Example 2. Isentropic fluid

The reference body is formed by macroscopically small elements of an isentropic flow of the perfect fluid.

It is well known that besides of traditional continual description, a perfect fluid can be considered as a collection of a finite number of identical macroscopic small particles that are under influence of interparticles forces which mimic the effect of pressure, viscosity etc [16], [21]. In particular, the fluid velocity in a given point is simply the velocity of the particle being at this point. At such description, the motion of the fluid particles is governed by solutions of ordinary differential equations of Newtonian or relativistic dynamics.

The motion of the fluid particles in an IRF is described by Lagrangian [38], [39] ${ }^{4}$

$$
L=-m c\left(G_{\alpha \beta} \frac{d x^{\alpha}}{d \lambda} \frac{d x^{\beta}}{d \lambda}\right)^{1 / 2} d \lambda
$$

where $\lambda$ is a parameter along 4-path of particles, $w$ is enthalpy per unit volume, $G_{\alpha \beta}=$ $\chi^{2} \eta_{\alpha \beta}$

$$
\chi=\frac{w}{\rho c^{2}}=1+\frac{\varepsilon}{\rho c^{2}}+\frac{P}{\rho c^{2}},
$$

$m c^{2}+\varepsilon$ is the rest internal energy, $\rho=m n, m$ is the mass of the particles, $n$ is the particles number density, $P$ is the pressure, $\varepsilon$ is the internal energy without and $\eta_{\alpha \beta}$ is the metric tensor in Minkowski space. According to (49), the line element of space-time in the comoving reference frame is given by

$$
d s^{2}=G_{\alpha \beta} d x^{\alpha} d x^{\beta} .
$$

The covariant derivative of tensor $G_{\alpha \beta}$ is equal to zero. Therefore, space-time in such $\mathrm{PRF}$ is Riemannian with the curvature is other than zero. The last description is equivalent to the one by the Lagrangian (65) [15].

\section{Example 3. Gravitational field}

Now we are ready to consider the case of gravitation.

An attempt to combine the theory of gravity in the Minkovski space with Einstein's theory was made in 1961 by Thirring in [8]. He suggested that gravity in Minkowsky space is described by a tensor function $\psi_{\alpha \beta}(x)$, and the motion of test particles is described by Lagrangian

$$
L=-m c\left[g_{\alpha \beta}(\psi) \dot{x}^{\alpha} \dot{x}^{\beta}\right]^{1 / 2} .
$$

This means that the metric tensor $g_{\alpha \beta}$ in Einstein's theory is a function of field $\psi_{\alpha \beta}(x)$. Taking this as a reasonable assumption, we will suppose that the motion of test particles in a gravitational field in inertial reference frames is described by the above Lagrangian. In this case, according to (49), the linear element $d s$ of the space-time is Riemannian in CRFs of the physical field $\psi_{\alpha \beta}$ existing in the Minkowski space.

\footnotetext{
4 This Result Will Be Discussed later in Detail.
} 
Thus, this result allows us to interpret the classical Einstein's gravitation equations as a description of gravity in the reference frames formed by particles moving in a certain classical gravity field $\psi_{\alpha \beta}(x)$ which exists in Minkowski space[5,6]. Of course, the problem of the existence of such correct equations of gravity is a separate task that is considered briefly in Section 5 .

In practice, the bimetrism considered here means the following.

We can assume that we are in an inertial reference frame to consider gravitation as a nonlinear physical field in Minkowski space. At the same time, we can consider ourselves to be in an PRF frame of reference of the mentioned field, where space-time is Riemannian.

The question: "Where is this inertial frame of reference" does not make sense, since the concepts of "geometry" and "frame of reference" by themselves, that is, separately, have no physical meaning.

Hence it follows that the correct equations of gravitation should be bimetric, as Rosen had long guessed [4]. However, now flat space-time has a physical meaning, and the relation of two types of gravity description is achieved in quite a definite way, namely, by a transition from one reference system to another. This is not a coordinate transformation. For this, e.g., the formalism developed in General Relativity [13] can be used. This question is apart from the objectives of this paper.

\section{The gravitational field as a tensor of rank 2}

So, our vacuum equations of gravitation have the form

$$
\nabla_{\alpha} B_{\beta \gamma}^{\alpha}-B_{\beta \delta}^{\epsilon} B_{\epsilon \gamma}^{\delta}=0
$$

In these equations, tensor $B_{\beta \gamma}^{\alpha}$ can be written as

$$
B_{\beta \gamma}^{\alpha}=D_{\beta \gamma}^{\alpha}-(n+1)^{-1}\left(\delta_{\beta}^{\alpha} D_{\gamma}+\delta_{\gamma}^{\alpha} D_{\beta}\right)
$$

where $D_{\alpha \gamma}^{\beta}=\Gamma_{\alpha \gamma}^{\beta}-\stackrel{\circ}{\Gamma_{\alpha \beta}^{\beta}}$ and $D_{\alpha}=D_{\alpha \beta}^{\beta}=\Gamma_{\alpha \beta}^{\beta}-\stackrel{\circ}{\Gamma^{\beta}}{ }_{\alpha \beta}$.

They are a system of differential equations for the geodesic-invariant tensor $B_{\beta \gamma}^{\alpha}(x)$ or for the functions $g_{\alpha \beta}(x)$. Since the equations are bi-metric, coordinate systems can be chosen arbitrarily in Minkowski space. From this equations, some equations for a function $\psi_{\alpha \beta}(x)$ can be obtained. They describe gravitation as a tensor field in Minkowski space.

The simplest way to obtain equations for $\psi_{\alpha \beta}$ is to assume that the tensor $D_{\beta \gamma}^{\alpha}=$ $\nabla^{\alpha} \psi_{\beta \gamma}$ where the operator $\nabla^{\alpha}$ is a covariant derivative in Minkowski space in the coordinate system used. As a result we obtain:

$$
B_{\beta \gamma}^{\alpha}=\nabla^{\alpha} \psi_{\beta \gamma}-(n+1)^{-1}\left(\delta_{\beta}^{\alpha} \nabla^{\sigma} \psi_{\sigma \gamma}+\delta_{\gamma}^{\alpha} \nabla^{\sigma} \psi_{\beta \sigma}\right)
$$

The identity $\mathrm{B}_{\alpha \gamma}^{\gamma}=0$ is satisfied as expected according to the definition of the Thomas symbols.

The object $\nabla^{\alpha} \psi_{\beta \gamma}$ is transformed in the same way as symbols $\Gamma_{\beta \gamma}^{\alpha}$ or $D_{\beta \gamma}^{\alpha}$ at geodesic mappings (2) because of its definition. Consequently, the mapping

$$
\nabla^{\beta} \psi_{\alpha \beta} \rightarrow \nabla^{\beta} \psi_{\alpha \beta}+(n+1) \varphi_{\beta}
$$


preserve the projectively invariant object $B_{\beta \gamma}^{\alpha}$, and therefore is a gauge transformation of $\psi_{\alpha \beta}$.

Obviously, the condition $\nabla^{\sigma} \psi_{\sigma \gamma}=0$ can be select a fix a calibration. In particular, we can select a convenient gauge condition $\nabla^{\sigma} \psi_{\sigma \gamma}=0$. At this conditions, eqs. (68) take the form

$$
\begin{aligned}
\nabla^{2} \psi_{\alpha \beta}-\nabla^{\sigma} \psi_{\alpha \gamma} \nabla^{\gamma} \psi_{\sigma \beta} & =0 \\
\nabla^{\sigma} \psi_{\sigma \gamma} & =0
\end{aligned}
$$

Properties of these equations have not been studied yet.

\section{The theory an observations}

\section{Motivation}

The previous reasoning seems very plausible. However, two questions arise:

1) The physical consequences of the modified gravitational equations do not contradict observational data?

2) Do the equations considered above lead to new results that can be verified?

Our attempt to realize the idea of the relationship of the space-time geometry and the reference frame used deserves the right to exist only in the case of a positive answer to these questions.

The motion of particles in a centrally symmetric field and the effects observed in this case were considered in $[11,12,14]$. It can be seen from these results that they practically coincide with the results of the Einstein model at distances from the center of gravity significantly exceeding the Schwarzschild radius of central mass. Here we will consider only effects that have not been sufficiently considered previously.

These equations lead to the same results that Einstein's equations at the distances $r$ from a gravity center which is much larger of the Schwarzschild radius $r_{g}$ of the central mass [11].

However, gravity properties become quite different when the distance approaches $r_{g}$. Instead of the classic Newtonian an expression for force $F$ acting on a rest test mass $m$ at rest we obtain:

$$
F=-\frac{m M G}{r^{2}}\left(1-\frac{r_{g}}{f}\right)
$$

where $r_{g}=2 G M / c^{2}$ is the Schwarzsschild radius of a dot mass $M$.

A singularity in the center is missing in this model. The gravitational force decreases within the Schwarzschild radius, and eventually tends to zero along with the distance from the center.

The weakness of the gravitational field inside the Schwarzchild radius leads to the possibility of the existence of cold stable supermassive objects without an event horizon, which are an alternative to supermassive black holes in the centers of galaxies[14,13, 11].

8.1 Gravitational force 
This modification of the classic Newtonian law is also consistent with recent observations.

Observation of gravitational waves during the merging of supermassive objects do not allow us to identify the nature of the objects.

The existence of supermassive objects without event horizon also does not contradict the result obtained with Event Horizon Telescope (EHT) collaboration observations [14] since the radius of the observed dark object is several times larger than their Schwarzschild radius. This remarkable

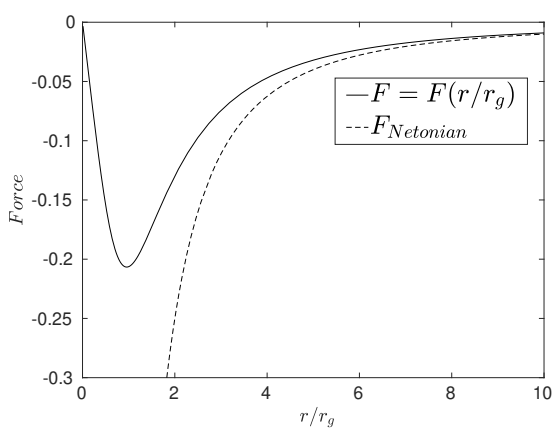

Fig. 2 The force acting rest-particle closes the Schwarzschild radius in arbitrary units. result demonstrates the existence of supermassive objects in the centers of galaxies, but it still does not make it possible to uniquely establish their nature. Greater accuracy is needed to establish the object mass[21] and which theory of gravity better describes the motion of light and particles in the vicinity of the Schwarzschild radius. The motion of light in the vicinity of the Schwarschild radius in the alternative theory was considered in [11].

In addition, we do not know whether the object is rotating or not. The equations of motion of particles and light in the vicinity of a rotating object are significantly different from the equations of motion of a static object. Therefore, further identification is necessary to identify the object.

It should be noted that an important result of the theory is also a quantitative explanation of the acceleration of the expansion of the Universe, which is impossible in the framework of General relativity. This will be shown briefly in this paper later.

8.2 Aceleretion of the Universe expansion as a consequence of gravity priperties

The considered gravitational equations lead to a natural explanation of the acceleration of the expansion of the universe as a manifestation of the properties of gravity $[5,6,14$, $13,7]$

The Lagrangian, which is invariant with respect to mapping $t \rightarrow-t$ and describes the motion of test particles in the the spherically symmetric field of a mass $M$ in Minkowski space has the form:

$$
L=m c\left[A(\mathrm{r}) \dot{r}^{2}+B(\mathrm{r})\left(\dot{\theta}^{2}+\sin ^{2} \theta \dot{\varphi}^{2}\right)-c^{2} C(r)\right]^{1 / 2}
$$

The solution of the equations (??) at the conditions

$$
\lim _{r \rightarrow \infty} A(r)=1, \lim _{r \rightarrow \infty}\left(B(r) / r^{2}\right)=1, \lim _{r \rightarrow \infty} C(r)=1
$$

is given by :

$$
\begin{array}{r}
C=1-r_{g} / f, f=\left(r^{3}+r_{g}^{3}\right)^{1 / 3}, B=f^{2}, \\
A=r^{4} / f^{4} C, r_{g}=2 G M / c^{2}
\end{array}
$$


It is easy to see that the main peculiarity of this solution is that functions $A, B$, an $C$ tend to their values in the Minkowski space ( $A=1, B=r, C=1$ ) at $r / r_{g} \rightarrow 0$, i.e., when the matter radius is much less then the Schwarzschild radius.

Consider when this condition can be satisfied.

Consider the force $F=m \ddot{r}$ ( or acceleration $\ddot{r}$ ) acting on a test particle of mass $m$ as a function of the distance $r$ from the observer. For a free radial motion of a test particle Lagrangian (72) is: $L=-m c\left(c^{2} C-A \dot{r}^{2}\right)^{1 / 2}$.

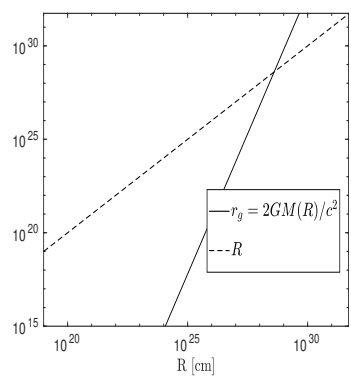

Fig. $3 r_{g}$ vs. the distance from an observer. It has the energy integral

$$
\frac{c C}{\left(c^{2} C-A \dot{r}^{2}\right)^{1 / 2}}=\bar{E}
$$

where $\dot{r}=d r / d t, \bar{E}=E / m c^{2}$ and $E$ is the energy of the particle. This equation gives the velocity $v=\dot{r}$ as a function of $r$ :

$$
v^{2}=c^{2} \frac{C^{4} f^{4}}{r^{4}}\left(1-\frac{C}{\bar{E}^{2}}\right)
$$

For parameter $\bar{E}$ three variants are possible: $\bar{E}>1, \bar{E}<1$ and $\bar{E}=1$. However, only $\bar{E}=1$ is consistent with observations.

Indeed, the distance to nearby galaxies satisfies inequality $r \gg r_{g}$ where $r_{g}=$ $2 G M / c^{2}$ and $M=4 / 3 \pi \rho r^{3}$. At this condition, $f \approx r$, and $C=1-r_{g} / r$. Consequently, we obtain from (76) that

$$
v=H r
$$

where

$$
H=\sqrt{(8 / 3) \pi G \rho}=1.8 \cdot 10^{-18} s^{-1}
$$

at $\rho=6 \cdot 10^{-30} \mathrm{~g} / \mathrm{cm}^{3}$. This is consistent with the observations.

If $\bar{E} \neq 1$, then equation (76) does not lead to the Hubble law since $v$ does not tend to zero when $r \rightarrow 0$. For this reason, we set $\bar{E}=1$.

With eq. (76) we can obtain a graph of the acceleration $a=v d v / d r$ as a function of the distance to a test particle.

It is very useful to compare the acceleration of a free test particle close a point mass $M$ and this value in the Universe close distance of $10^{28} \mathrm{~cm}$., which are given in fig 3 and 4 . 


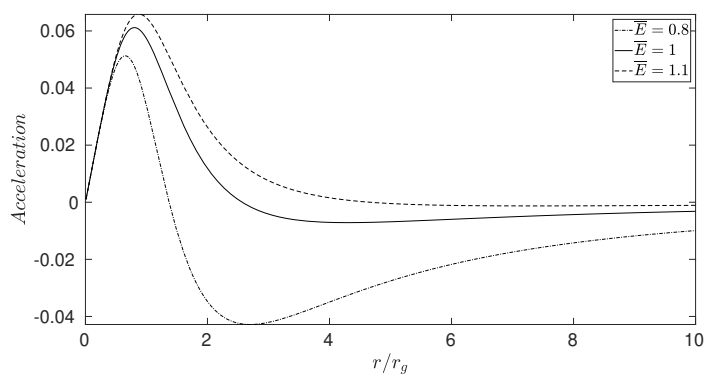

Fig. 4 Acceleration (arbitrary units) of free-falling test particles to a central point mass $M$ as the function of the distance from the mass in the Schwarzschild radius's. The value $\bar{E}$ is the dimensionless energy $E / m$.

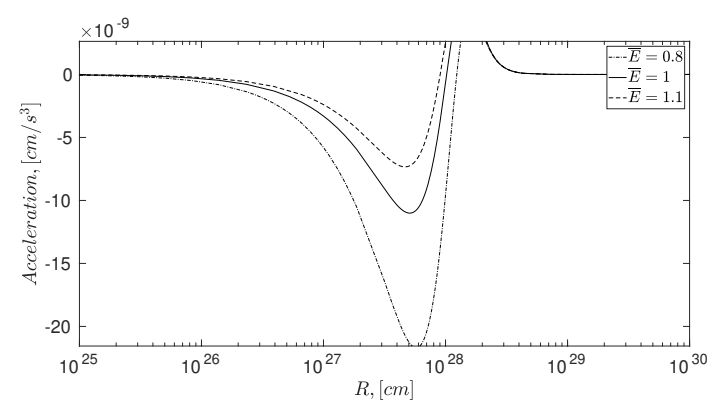

Fig. 5 Acceleration of test particles in the Universe at the distance vicinity of $R=10^{28} \mathrm{~cm}$.

A comparison of graphs 3 and 4 shows that they have similar features, since in both cases, accelerations are considered in the area where the equality $r / r_{g}=1$ holds.

The Schwarzschild radius $R_{g}$ becomes more than $R$ at $R>1.5 \cdot 10^{28} \mathrm{~cm}$. Before this, at $R=6 \cdot 10^{27} \mathrm{~cm}$, the acceleration change sign, in analogy with the particle free falling to a point mass (fig 2). If $R>6 \cdot 10^{27} \mathrm{~cm}$, the acceleration is positive. Hence, for sufficiently large radius $R$ the gravitational force gives rise to an acceleration of remote galaxies.

This is a natural explanation of the observed acceleration of the expanding Universe, which is confirmed by the satisfactory coincidence of the theoretical and the real Hubble diagram [5]. Besides, in the next section, additional justification for this statement provides by calculating the deceleration parameter.

\subsubsection{Deceleration parameter}

The calculation of the deceleration parameter is an important method for checking the relativistic expression used above for the speed and acceleration of a test particle in the expanding Universe.

The acceleration as a function of the distance is $g=v^{\prime}(r) v(r)$ where the velocity $v$ is given by (76). It allows us to find the value of the deceleration parameter: 


$$
q=-\frac{g \cdot r}{v^{2}} .
$$

The fig. 6 shows $q$ as a function of the distance of an object from the observer.

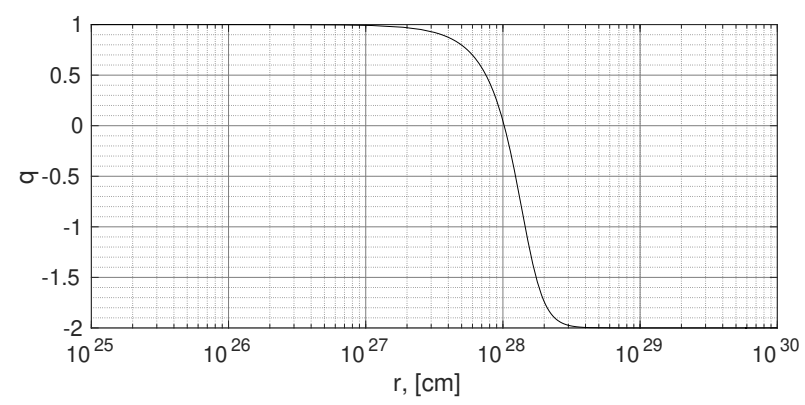

Fig. 6 Parameter $q$ vs. distance to the object.

This graph shows that the observed value of present-day $q_{0} \approx-0.55$ [15] corresponds to the geometrical distance of the remote objects of about $10^{28} \mathrm{~cm}$. It is consistent with fig. 4 that shows the acceleration of distance objects.

8.3 The energy of the gravitational field

In the Einstein theory and its modifications, which are based on the identification of gravity and curvature of space-time, the strict meaning of gravitational energy is not clear. However, a correct definition of the gravity energy is necessary to find gravitational binding energy of the Universe. Such a definition is possible in the Minkowski space.

Einstein's vacuum equations coincide with eq. (25) at the gauge condition $Q_{\alpha}=0$. Therefore, following Weinberg [14], we can suppose that the nonlinear term of the Einstein equations is associated with gravitational energy. To be more precise, we assume that equations ( 25 can be written as:

$$
B_{\alpha \beta ; \gamma}^{\gamma}=k t_{\alpha \gamma}
$$

where the energy-momentum tensor of the field is $t_{\alpha \gamma}=k^{-1} B_{\alpha \delta}^{\epsilon} B_{\beta \epsilon}^{\delta}$ and $k$ is some constant.

It is not hard to show that this assumption makes sense.

We use the previously mentioned expression for the gravitational force in a spherically symmetric gravitational field, which follows from the gravity equations used here

$$
F=-\frac{m M G}{r^{2}}\left(1-\frac{r_{g}}{f}\right)
$$

for a spherically symmetric field.

We define the potential of the force $F$ as $U(r)=\int_{0}^{r} \frac{F\left(r^{\prime}\right)}{m} d r^{\prime}$.

Double differentiation of function $U(r)$ gives 


$$
U^{\prime \prime}=-\frac{2 U^{\prime}}{r}-\frac{2 G^{2} M^{2}}{c^{2} f^{4}}
$$

Now denote

$$
t_{00}=\frac{G M^{2}}{2 \pi f^{4}} .
$$

As a result, we obtain that function $U(r)$ satisfies the differential equation

$$
\nabla^{2} U=-4 \pi \bar{\rho}
$$

where $\bar{\rho}=t_{00} / c^{2}$.

This equation has a clear physical meaning. This is the Poisson equation for spherically symmetric field, in which $\bar{\rho}$ is the mass density of the gravitational field created by the mass $M$. In the absence of another matter, only the density of the gravitational field can lead to the fact that the potential will differ from zero.

This result shows clearly that $t_{00}$ is the density of the gravitational field in the considered theory where the gravitational force is given by eq. ( 78 ).

\subsubsection{Test}

The resulting expression for the energy-momentum tensor withstands the most difficult test - the calculation of the field energy of a point mass.

It follows from above equations that zero-component $t_{00}$ of the energy-momentum tensor of a point mass $M$ is given by

$$
t_{00}=\varkappa^{-1} \frac{r_{g}^{2}}{2 f^{4}}
$$

which gives, when integrating over entire space with $\varkappa=4 \pi G / c^{4}$

$$
\int t_{00} d V=M c^{2}
$$

and $\int t_{0 i} d V=0$ for $i=1,2,3$.

Thus the energy of a point mass is finite and equal to the expected value.

Besides, it should be noted also that the integration of the density $t_{00}$ over the volume of the visible Universe (radius $R \sim 10^{28} \mathrm{~cm}, \rho \sim 6 \cdot 10^{-30} \mathrm{~g} / \mathrm{cm}^{2}$ ) gives the correct value of its mass $\sim 10^{56} \mathrm{~g}$.

For the above reasons, we can consider the expression (80) as the energy density of the gravitational field of a mass $M$.

\section{Gravitational mass-energy defect as a reason of the hot Universe}

For this reason, we can consider (80) as the density energy of the gravitational field of the mass $M$.

It follows from (80) that the energy $\int_{0}^{R} t_{00} d V$ in the volume inside radius $\mathrm{R}$ around the central mass $M$ is 


$$
\mathcal{E}_{\mathrm{sph}}=M \mathrm{c}^{2}\left[1-\frac{\mathrm{r}_{\mathrm{g}}}{\mathrm{f}}\right]
$$

where $f=\left(r_{g}^{3}+r^{3}\right)^{1 / 3}$ and $f=\left(r_{g}^{3}+r^{3}\right)^{1 / 3}$. The total energy is $E_{\mathrm{sph}}+\mathcal{E}_{\text {out }}$ where $\mathcal{E}_{\text {out }}=\int_{\mathrm{R}}^{\infty} t_{00} d V=M c^{2} \frac{r_{g}}{f}$, so that $\mathcal{E}_{\text {out }}+E_{\text {sph }}=M c^{2}$.

Since the solution of the field equations under consideration is refer to any spherically symmetric mass, it implies that the above formulas give the gravitational energy of any spherically symmetric mass $M$ with radius $R$.

Expression (81) is the difference of the two terms. The second term is the gravitational binding energy $E_{b}=M c^{2} r_{g} / f$.

The internal energy of the gravitational field of the visible region of the Universe is $E=E_{0}-E_{b}$ where $E_{0}=M_{0} c^{2}$ is the energy of a newborn mass $M_{0}$ and $E_{b}$ is the gravitational binding energy at radius $R$.

According to (81),

$$
E=E_{0}\left(1-\frac{R_{g}}{\left(R_{g}^{3}+R^{3}\right)^{1 / 3}}\right)
$$

where $R_{g}=2 G M_{0} / c^{2}, M_{0}=E_{0} / c^{2}$. The quantity $E_{b}=E_{0} R_{g} / f$, where $f=$ $\left(R_{g}^{3}+R^{3}\right)^{1 / 3}$, is the gravitational binding energy of the mass inside radius of $R$.

It can be seen from (82) that the internal energy of the region inside $R_{g}$ can be zero only in the case when the radius of matter tends to zero. Only in this case, a point mass of the newborn Universe satisfies the law of energy conservation.

Fig. 6 shows the graph the internal energy $E / c^{2}$ as the function of the radius $\mathrm{R}$ of the matter for the two magnitudes of $E_{0}$ which are close to the energy $M_{0} c^{2}$ of the observed Universe. This graph can be considered as the dependence of the internal energy of the expanding Universe on its radius in the past in the most interesting interval of $R$.

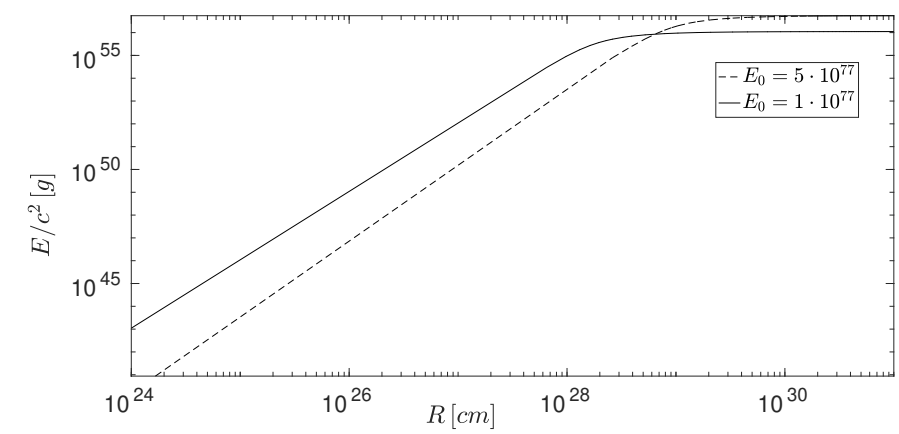

Fig. 7 Mass-energy vs. radius $\mathrm{R}$ of the Universe.

The graph shows that the increase in mass ends at a certain radius of matter, which indicates its size since the density here must rapidly decrease. This implies that the gravitational binding energy determines the size of a self-gravitating matter.

A spontaneous appearance of mass $M_{0}=E_{0} / c^{2}$ causes the immediate appearance of the gravitational binding energy (mass-energy defect), whose release is the necessary condition of the appearance $M_{0}$ as a real object. 
It is very important that the model under consideration can be compared with observations since we know the density $\varrho_{\gamma}$ of cosmic microwave radiation and the corresponding redshift. The density is $\varrho_{\gamma}=2 \cdot 10^{-13} \mathrm{erg} / \mathrm{cm}^{3}$, and redshift is $z \approx 10^{3}$.

Figure 7 shows the dependence of the radiation density $E_{b} / V$ in the volume $V$ due to the gravitational mass-energy defect on the radius $R$ of the expanding Universe.

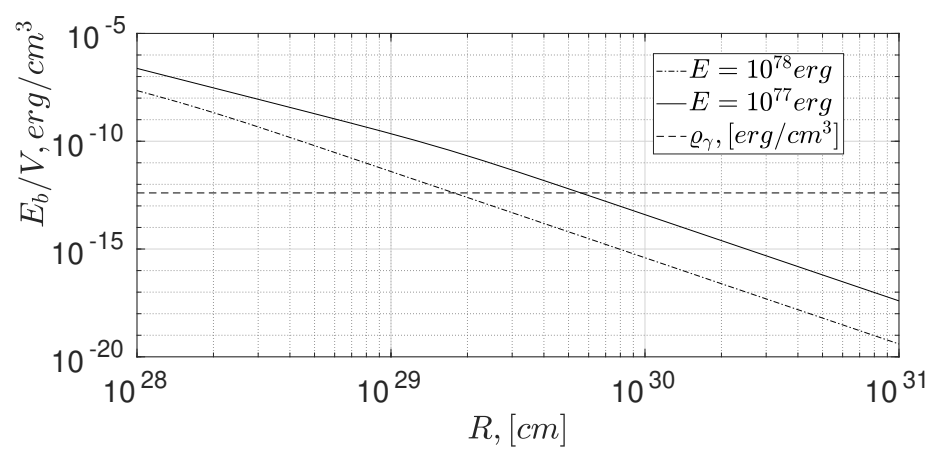

Fig. 8 The energy density of the radiation as the function of the Universe radius.

We see that the radiation density due to the gravitational mass-energy defect is $2 \cdot 10^{-13} \mathrm{erg} / \mathrm{cm}^{3}$ at the radius $\sim 5 \cdot 10^{29} \mathrm{~cm}$.

This distance is consistent with the graph in Fig. 4 where the acceleration of the particles is shown.

In addition, we can also find the relationship between the geometric distance from the observer and the redshift $z$. It is obtained with a formula obtained in [5]:

$$
z=\left(C(r)^{-1} \frac{1+v(r) / c}{1-v(r) / c}\right)^{1 / 2}-1
$$

where $C(r)=1-r_{g} /\left(r_{g}^{3}+r^{3}\right)^{1 / 3}$ and $v(r)$ is the recession velocity in Minkowski space at zero-density and pressure.

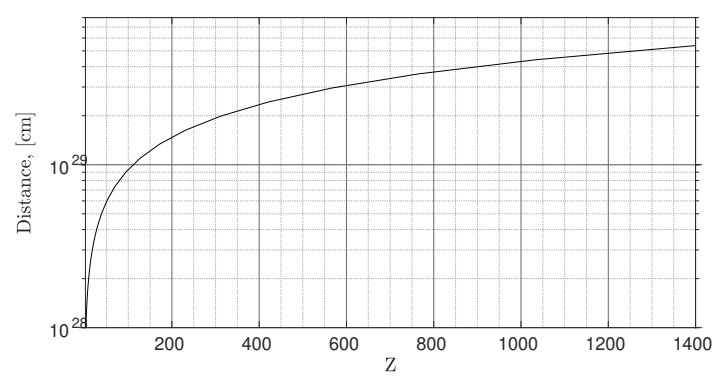

Fig. 9 R vs. $z$

It can be seen from figure 8 that the distance $r \approx 5 \cdot 10^{29} \mathrm{~cm}$ corresponds to the $z=10^{3}$ which is CMBR density. This is consistence with observations. 


\section{Direct evidence for inflation}

Although the model of inflationary cosmology is almost generally accepted, the nature of the inflanton remains unclear. Therefore, it is not surprising that attempts have been made to explain inflation without the need to use hypothetical fields, and to obtain inflation as a consequence of the properties of space-time in modified versions of the Einstein equations $[17,21,16,19,18]$. However, despite this, the problem cannot be considered solved.

However, the triggering of inflation could also be the result of a gravitational massenergy defect in the newborn Universe.

Let us find the dependence of the radius of the spherically symmetric mass $E / c^{2}$ on time during its expansion, due to the gravitational mass-energy defect.

Consider a test particle located on the edge of the expanding spherical mass $M$. The desired dependence is determined by the change in the force acting on this particle by the substance of the mass $M$ in the process of the sphere expansion.

External matter does not affect this due to the supposed homogeneity and isotropy of the Universe, as well as due to the existence of the gravitational event horizon.

To avoid doubts about the correctness of the result obtained by numerical methods, we consider the solution of this problem in two different ways.

1. The Lagrangian for the motion of a particle during its radial expansion of mass $M$ in Mikowski space has the form (72)

$$
L=-m c\left(C c^{2}-A \dot{r}^{2}\right)
$$

where $C$ and $A(74)$ are the functions of the distance $r$ from the mass center. The corresponding particles motion equation is

$$
\ddot{r}=-c^{2} \frac{C^{\prime}}{A}+\left(\frac{C^{\prime}}{C}-\frac{A^{\prime}}{2 A}\right) \dot{r}^{2} .
$$

Sometimes, the solution of this equation it is easy all obtained by the expression for the velocity ( 76) which follows from an integral of motion considered in section 2 :

$$
\dot{r}^{2}=c^{2} \frac{C^{4} f^{4}}{r^{4}}\left(\frac{r_{g}}{f}\right) .
$$

The speed and acceleration do not have a singularity in the mass center, they tend to zero together with distance $r$. The dependence of $r$ on $t$ can be found both for the expansion of mass from zero radius, and for history back in time, starting from the radius of the visible Universe was approximately $1 \cdot 10^{-100} \mathrm{~cm}$. Figure 9 shows this solution.

2. Of course, the above method does not allow us to take into account the influence of pressure, since we are dealing with a dust-like model of the Universe

The expansion of the Universe as a function of time, taking into account pressure inside the mass $M$, can also be obtained by another method. 


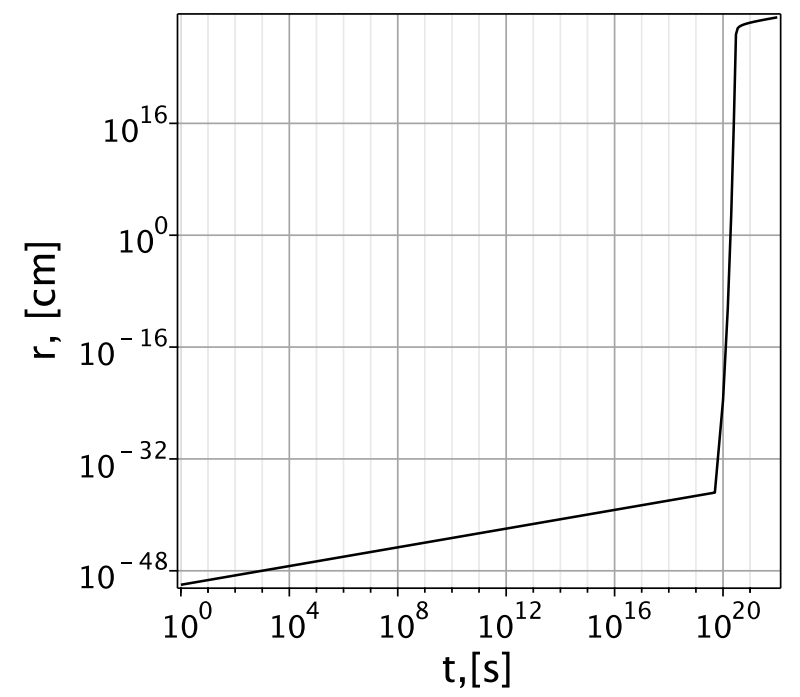

Fig. 10

It was shown in [15] that the motion of macroscopically small elements of an isentropic fluid ("particles") of a mass $m$ is described by the following Lagrangian ${ }^{5}$,

$$
L_{f l}=-m c \chi\left(\eta_{\alpha \beta} d \dot{x}^{\alpha} d \dot{x}^{\beta}\right)^{1 / 2}
$$

where $\eta_{\alpha \beta}$ is metric tensor of Minkowski space in coordinate system used,

$$
\chi=\frac{w}{\rho c^{2}},
$$

$w$ is the enthalpy per unit volume, $\rho=m n, n$ is the particle number density.

This means that macroscopically small elements of the isentopic fluid move along the geodesic lines of the Riemannian space with a line element $d s=(m c)^{-1} d S$ where $S=\int L_{f l}(x, \dot{x}) d t$,i.e.,

$$
d s=\left(G_{\alpha \beta}(x) d x^{\alpha} d x^{\beta}\right)^{1 / 2}
$$

where $G_{\alpha \beta}=\chi^{2} \eta_{\alpha \beta}$.

This space-time is conformal to the Minkowski one.

This result allows us to study the properties of a fluid by studying the motion of its elements instead of studying the streamlines obtained by solving the complex relativistic Euler equations.

For a radial motion in the expanding Universe in Minkowski space we have:

5 This approach is inspired by the existence of an effective numerical solution of problems of hydrodynamics [16], known as Smoothed Particle Hydrodynamics (SPH). In this method, a fluid is considered as composed by finite number of particle. These particles move under the action of inter-particle forces which mimic effects of pressure, viscosity, and so on. Due to the replacement of integration by summation over number of particles, continual derivatives become the time derivative along the particle trajectory, and as a result, the motion of particles governed by ordinary differential equations of classical mechanics. 


$$
L_{l f}=-m c \chi(r)\left(c^{2}-\dot{\mathrm{r}}^{2}\right)^{1 / 2}
$$

The enthalpy of a fluid is $W=E+p V$, and $w=E / \rho c^{2}+p$ where $\mathrm{p}$ is the pressure.

Using equality equality $M_{0}=\rho V=$ Const we obtain fro (82) that

$$
\chi=1-\frac{R g}{f}+\frac{P}{\rho c^{2}}
$$

and the above Lagrangian yields the following equation of the radial motion of "particles" at the Universe expansion:

$$
\ddot{r}=-c^{2} \frac{\chi^{\prime}(r)}{\chi(r)} \cdot\left(1-\frac{\dot{r}^{2}}{c^{2}}\right) .
$$

In order to solve this equation, we can sometimes use an integral of the motion

$$
\frac{m c^{2} \chi}{[1-(\dot{x} / c)]^{1 / 2}}=\text { Const }
$$

that yields the following equation of the motion of macroscopic small element ("particle") of the isentropic fluid

$$
\left(\frac{\dot{r}}{c}\right)^{2}=1-\frac{\chi^{2}}{\bar{E}^{2}}
$$

where $\bar{E}=E / m c^{2}$ and $E$ is the energy of the "particle".

Since Hubble's law in the absence of pressure must be satisfied, the constant $\bar{E}$ must be equal to 1 in vacuum.

Below graphs of the solutions $r=r(t)$ of equation ( 86) are given at $p=0$.

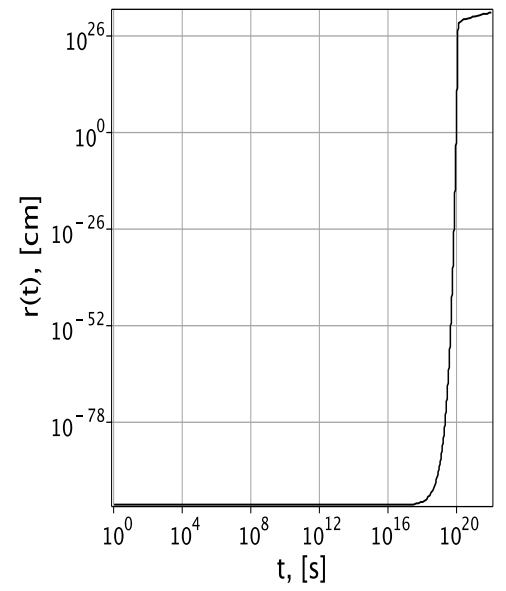

Fig. 11 The Universe radius $r(t)$ starting for the initial conditions: $r(0)=$ $10^{-100} \mathrm{~cm}$. and $\dot{r}(0)=0$.

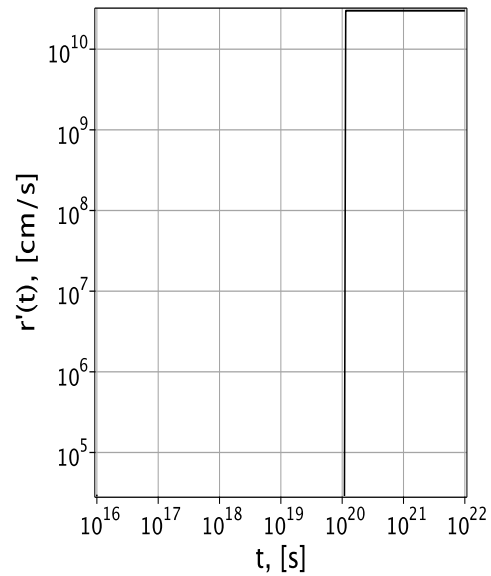

Fig. 12 The Universe velocity $\dot{r}(t)$ for the initial condition: $r(0)=10^{-100} \mathrm{~cm}$. and $\dot{r}(0)=0$. 
It should not be thought that there was a singularity before the exponential expansion, since all the equations of motion of the test particles give zero velocity when radius of the Universe tends to zero.

It should be noted that the above-obtained graphs of evolution of the radius of the expanding universe can be obtained in another way. The solution (74) of equations (40), in Minkowski space, which is similar to the Schwarzschild sulution in GR, allows you to get the distribution of velocities $v(r)$ and accelerations $w=v d v / d r$ at an arbitrary radius $r$. The solution of the differential equation $d r / d t=v(r)$ with the initial condition $r(0)=r 0$ close to $r 0=0$ gives the evolution of the geometric radius of the visible universe. It is similar to that obtained on the graph above.

10.1 The time and energy before inflation

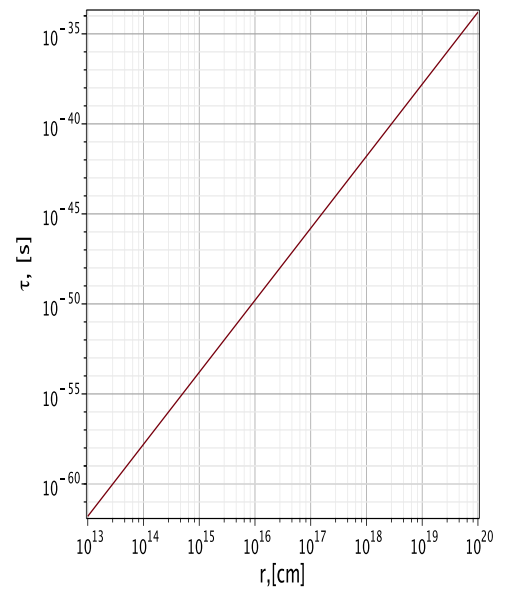

Fig. 13 The proper time $\tau$ vs. radius $r$.

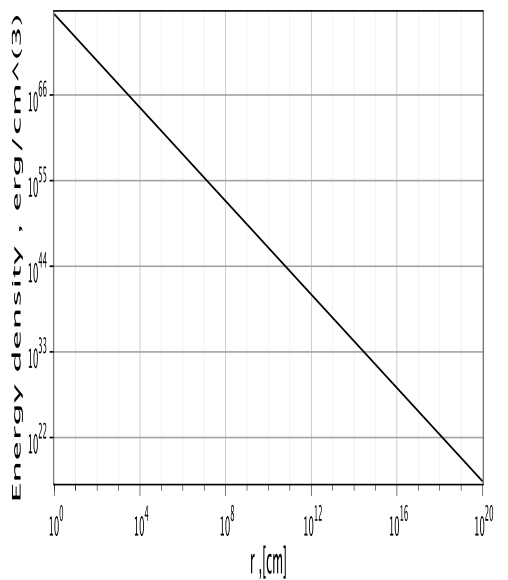

Fig. 14 The energy density $E_{b}$ vs. radius of the expanding Universe.

The purpose of this paper is to show that the gravitational mass-energy defect is a likely cause of the Big Bang energy.

There are at least two consequences of the considered model, which can help further study the structure of the substance before inflation and study its results.

1) Time $t$ on the graphs is not of course physical time. Physical time is measured by the length of its own world line of particle motion. When the particles of the newborn Universe move radially, the proper time is $\tau=d s / c$ where $d s$ is the line element

$$
d s=C-A\left(\frac{v}{c}\right)^{2}
$$

where values $A, C$ and the velocity $v$ are given above in this Section. The dependency $\tau$ on $t$ is given by fig. 12 .

It is very interesting that these results are consistent with well-known results obtained on the basis of General Relativity, except for the absence of singularity at the time of the birth of the Universe. 
2) In the model of the dynamics of the universe considered here, the energy of the newborn Universe is quantity determined by the Schwarzschild radius $R_{g}$, which in turn is given by the average density $\rho$ of the visible Universe.

The energy released is the gravitational binding energy, which, unlike GR, can be found for any radius $r$ of the

Figure 13 shows the dependence of the density of released energy on the radius of the Universe on the radius $r$ before inflation. This is important information for assumptions about the structure of matter, from the Planck era to the time of inflation.

\section{Conclusion}

The idea of the relativity of space-time geometry relative to the properties of measuring instruments is difficult to understand. It has never been realized in physics and leads to a profound revision of the foundations of the theory of gravitation. Only future, more detailed observations can confirm or reject it.

\section{References}

1. H. Reichenbach, Phylosophy of space and Time, New York, (1958)

2. L. Eisenhart, Riemannian geometry, (Princeton, Univ. Press) (1950)

3. A. Petrov, Einstein Spaces, (New-York-London, Pergamon Press. (1969)

4. N. Rosen, Gen. Relat. Grav., 4, 435 (1973)

5. W. Thirring, Ann. Phys., 16, 96 (1961)

6. L. Verozub, Phys. Lett. A, 156, 404 (1991)

7. L. Verozub, AIP Conf. Proc., 624, 32 (2002)

8. L. Landau, and E. Lifshitz, The Classical Theory of Field, (Massachusetts, Addison Wesley) (1971)

9. H.Rund, The differential geometry of Finsler space, (Berlin-Göttingen-Heidelberg, Springer), (1959)

[4] Landau, L.; Lifshitz, E. , The Classical Theory of Fields, 4th. Revised ed.; Pergamon, Oxford.

[5] L. Verozub, Annalen der Physik, 16, No. 1, p. 28, (2008)

[6] L. Verozub, Space-time relativity and gravitation, Lambert Acad. Publ. (2017)

[7] N. Rosen, Gen. Relat. Grav., 4, 435 (1973)

[8] W. Thirring, Ann. Phys., 16, 96 (1961)

[9] L. Eisenhart, Non-Riemannian geometry, Dover Publ.Inc. (2005)

[10] A. Petrov, Einstein Spaces, (New-York-London, Pergamon Press. (1969)

[11] L. Verozub, Astron. Nachrichten 317, 107 (1996).

[11a] New Trends in Theoreticak and Observational Cosmology, Eds K. Sato \& T. Shiromizu, Universal Acad. Press, Tokyo (2002)

[12] L. Verozub and A. Kochetov, Astron. Nachrichten 322, 143 (2001).

[13] L. Verozub, Nuovo Cimento,123,1653 (2008).

[14] L. Verozub, Astronomische Nachrichten, 327, No. 4, p. 355, 2006

[15] L. Verozub, Int.J.Mod.Phys.D, $2008 \mathbf{1 7} 337$

[38] .L. Verozub, Int. J.Mod.Phys.D, 17, 337 (2008)

[39] L. Verozub, Grav. and Cosm., 19, 124 (2013)

10. J. Mikeš, V. Kiosak, A. Vanšurová, Geodesic mappings, Olomouc (2008)

11. T. Thomas, The differential invariants of generalized spaces, (Cambridge, Univ. Press) (1934).

12. O. Veblen, Quart. Journ. Math., 1, 66 (1930)

13. V. Antonov, V. Efremov, \& Yu. Vladimirov, Gen.Relat. Grav., 9, 9 (1978)

14. S. Weinberg, Gravitation and Cosmology, (New-York-London-Sydney-Toronto, J. Wiley \& SonsInc.) (1972)

[21] K. Kalmet and C. Fritz, Phys. Lett. B,747(C), (2015)

15. K. Kalmet and C. Fritz, Phys. Lett. B,747(C), (2015) 
16. P.Kanti, R.Gannouji and N. Dadlich, Phys./Rev. D, 92, 041302 (2015)

17. F.Florini, R.Ferrero, Phys.Rev. D,75, 084031 (2007)

18. S.Tsujikavwa, R. Maartens, R.Brandenberger, Phys.Lett. B, 574, (2003).

19. F. Melia, A\&A, 553, A76 (2013)

[14] H. Falcke, et al.,Astrophys.Letters,875,N1,April 10 (2019)

[21] L., Titarchk et al, AandA,633,A73 (2020)

[15] Jones, H.; Lambourne, Robert J. , An Introduction to Galaxies and Cosmology. Cambridge Univ. Press. p. 244. (2004).

20. S., Weinberg, Gravitation and Cosmology, Wiley (1972 F. Tipler, Mon. Not.R. Astron. Soc., 282, 206 (1996)

[50] L. Abrams, Phys.Rev., 20, 20 (1980)

[16] J. Monaghan, Ann.Rev.Astron.Astrophys. 199230 543;

[21] J. Monaghan and D. Rice, Mon.Not.R.Astron.Soc. 2001328381

[51] K. Schwarzschild, d. Berl. Akad., 189 (1916) 